\title{
Acute neurological deterioration after surgical interruption of spinal dural arteriovenous fistulas: clinical characteristics, possible predictors, and treatment. Patient series
}

\author{
Akihiko Saito, MD, PhD, Naoki Yajima, MD, PhD, Kimihiko Nakamura, MD, PhD, and Yukihiko Fujii, MD, PhD \\ Department of Neurosurgery, Brain Research Institute, Niigata University, Niigata, Japan
}

BACKGROUND Acute neurological deterioration develops paradoxically in some patients after obliteration of a spinal dural arteriovenous fistula (SDAVF), with thrombosis of the spinal cord veins as its primary cause. The authors aimed to clarify the clinical and radiological characteristics of acute deterioration to identify high-risk patients. They also discussed the optimal treatment for this complication.

OBSERVATIONS Ten patients with SDAVF presenting with congestive myelopathy who received microsurgical interruption were retrospectively reviewed. Severe myelopathy developed in three patients on postoperative days 1 to 3 . Anticoagulation therapy was effective; however, discontinuing anticoagulants under residual spinal cord congestion caused redeterioration. These patients were characterized by significantly extended transit time on angiography and significant prolongation of spinal cord congestion. Acute deterioration exhibited a strong correlation with transit time (coefficient, $0.825 ; p=0.006$ ) and a strong correlation with spinal cord edema before surgery (coefficient, $0.656 ; p=0.040$ ).

LESSONS Acute deterioration after SDAVF treatment is likely to develop in patients with severe venous outflow impairment. Its pathology is prolonged spinal cord congestion caused by postoperative venous thrombosis and preexistent severe venous outflow impairment. Anticoagulation treatment should be continued for patients with acute deterioration until the resolution of spinal cord congestion is confirmed with magnetic resonance imaging.

https://thejns.org/doi/abs/10.3171/CASE21548

KEYWORDS spinal dural arteriovenous fistula; congestive myelopathy; surgical interruption; venous thrombosis

Venous hypertension of the spinal cord is a known cause of progressive myelopathy associated with spinal dural arteriovenous fistula (SDAVF). ${ }^{1-3}$ Currently, the predominant view is that in addition to the existence of arteriovenous shunt, a decrease in venous outflow from the spinal cord plays an essential role in venous hypertension development. ${ }^{4-6}$ Radicular veins decrease in number with the progression of fibrosis and thrombosis related to aging. ${ }^{4-6}$ Although the pathomechanism of arteriovenous shunt formation remains unclear, once a fistula has formed within the dura, various pathological changes, including fibrosis, hyalinization, wall thickening, and microthrombosis, occur in the spinal cord veins. ${ }^{1,7,8}$ It has been speculated that sclerotic changes in the spinal cord veins, accelerated by arteriovenous shunt, further exacerbate the venous outflow of the spinal cord. ${ }^{4-6}$ Because of the lack of valves, increased pressure in the perimedullary veins is directly transmitted to the intramedullary veins, leading to congestive edema of the spinal cord and decreased tissue perfusion pressure, which cause progressive spinal cord dysfunction. ${ }^{1-6}$

Since the pathophysiology and microvascular anatomy of SDAVFs have been clarified, eliminating spinal cord congestion by arteriovenous shunt obliteration has been recognized as the treatment goal. ${ }^{9,10}$ Most patients improve or stabilize after spinal cord congestion is eliminated by microsurgical interruption of the fistula or endovascular embolization. ${ }^{11-16}$ However, some patients were reported to develop acute neurological deterioration despite successful obliteration of the fistula, regardless of the therapeutic

ABBREVIATIONS $\mathrm{AC}=$ anticoagulation therapy; $\mathrm{CT}=$ computed tomography; $\mathrm{CVJ}=$ craniovertebral junction; DSA $=$ digital subtraction angiography; $\mathrm{HIA}=$ hyperintensity area; MRI = magnetic resonance imaging; SDAVF = spinal dural arteriovenous fistula.

INCLUDE WHEN CITING Published December 20, 2021; DOI: 10.3171/CASE21548.

SUBMITTED October 19, 2021. ACCEPTED October 25, 2021.

(C) 2021 The authors, CC BY-NC-ND 4.0 (http://creativecommons.org/licenses/by-nc-nd/4.0/). 
modality. ${ }^{17-24}$ To date, progressive thrombosis of the spinal cord veins after shunt obliteration is considered its main cause. ${ }^{25}$ Based on this hypothesis, several authors have reported the efficacy of antithrombotic therapy, using an anticoagulant or a substitute for its treatment. $^{19-22}$ However, the clinical presentations, radiological characteristics, and predictors of acute deterioration currently remain unclear. Furthermore, the optimal duration of anticoagulation therapy has not yet been established. The primary objective of this study was to clarify the clinical presentation and radiological characteristics of patients who presented with acute deterioration to identify high-risk patients. In addition, we explored the optimal treatment of this complication, which may develop after successful fistula obliteration.

\section{Study Description}

\section{Patient Selection and Clinical Evaluation}

We retrospectively analyzed the medical data of 10 patients who underwent microsurgical interruption of SDAVF presenting with congestive myelopathy. Gait and micturition dysfunction at presentation and at 1 year after surgery were assessed based on the AminoffLogue Scale (ALS) score. ${ }^{26}$ According to the postoperative clinical course, we divided the 10 patients into two groups: Group A (patients with acute deterioration; $n=3$ ) and Group B (those without acute deterioration; $n=7$ ).

\section{Radiological Evaluation}

The diagnosis was verified with whole spinal magnetic resonance imaging (MRI) and digital subtraction angiography (DSA) in all patients. A computed tomography (CT) scan was obtained for all patients on postoperative day 1 to investigate surgical complications, and postoperative DSA was performed during the follow-up period to confirm the surgical result.

We evaluated the degree of venous outflow obstruction using preoperative DSA. In SDAVF, venous drainage impairment from the spinal cord is most significant at the fistula site. ${ }^{25}$ Therefore, we measured the time in which the contrast medium passed through the coronal venous plexus between one vertebral level above and below the reflux point.

To evaluate the chronological changes in spinal cord congestion, follow-up MRIs were obtained for all patients 3, 6, and 12 months after surgery. We assessed the craniocaudal extent of the intramedullary hyperintensity area (HIA) on sagittal T2-weighted MRI by counting the adjacent vertebral bodies. The chronological changes in the intramedullary HIA were represented by percent changes from the preoperative baseline.

\section{Surgical Treatment}

We performed microsurgical interruption via targeted laminectomy involving division of the intradural arterialized draining vein and coagulation of the fistula. Normalization of the arterialized perimedullary veins was confirmed by Doppler sonography and indocyanine green fluoroscopy or intraoperative DSA, if needed. For the lesion at the craniovertebral junction (CVJ), interruption was performed via ipsilateral small lateral suboccipital craniectomy and C1 hemilaminectomy.

\section{Anticoagulation Therapy}

In this series, no patient, except 1 , received postoperative prophylactic heparinization. Only Patient 3 in Group A received continuous intravenous administration of heparin (5,000 IU/day), starting from postoperative day 1 . When acute neurological deterioration developed, continuous intravenous administration of therapeutic heparinization $(15,000$ to 20,000 IU/day) was initiated for assumed venous thrombosis after an emergency MRI or CT scan was obtained. Therapeutic heparinization was continued for 7 to 10 days to maintain the activated partial thromboplastin time at 1.5 to 2.0 times the control value. An oral anticoagulant, warfarin potassium, was prescribed after heparinization at an internalized normalized ratio maintained between 2.0 and 3.0. The duration of oral anticoagulant administration for each patient was determined on a trial basis.

\section{Statistical Analyses}

We compared the groups in terms of patient age, duration of symptoms, preoperative and postoperative ALS scores for gait and micturition, transit time of the contrast medium, craniocaudal extent of intramedullary HIA on preoperative T2-weighted MRI, and percent changes in intramedullary HIA using Student t-test.

The correlation between the development of acute deterioration and clinical characteristics such as age, duration of symptoms, preoperative ALS scores, transit time, and extent of intramedullary HIA on preoperative MRI were tested with Spearman's rank correlation to detect possible predictors of acute deterioration.

We assessed the standardized effect size, which is not affected by the sample size, by calculating Cohen's $d$ for the Student t-test results; in addition, we performed a post hoc analysis using G*Power 3.1 to evaluate the statistical power of all results. The value of Cohen's $d$ is defined as follows: $d=0.2$, small; 0.5 , medium; 0.8 , large; and $>1.0$, extremely large effect size. We considered $p$ values $<0.05$ to indicate statistical significance. A power value of 0.95 $(1-\beta)$ indicates strong statistical power under a significance level set at 0.05. We performed all statistical analyses using BellCurve for Excel version 3.20 (Social Survey Research Information Co.).

\section{Patient Characteristics and Surgical Results}

The clinical and radiological data of patients in this study are summarized in Table 1 . This study comprised 10 patients $(9$ men and 1 woman) with a mean age of 58.7 years (range, 39 to 77 years). All patients presented with slowly progressive congestive myelopathy. Fistula sites on DSA were thoracic in 6 patients, sacral in 2, lumbar in 1, and at the CVJ in 1. In one of the cases with sacral lesions, a small fistula existed epidurally accompanied by intrathecal venous drainage alone. This case was added to this study because its pathophysiology and surgical strategy have been pointed out to be similar to those of SDAVF. ${ }^{27}$ No multiple fistulas were encountered in this study.

Five patients were primarily treated with surgery, and others were treated with surgery after unsuccessful endovascular embolization. Surgical complications included delayed subcutaneous cerebrospinal fistula in Patient 2 assigned in Group A, treated by lumbar drainage. There were no hemorrhagic complications related to anticoagulation therapy (AC). Postoperative DSA was performed at a mean $169 \pm 100$ days after surgery in Group $A$ and at a mean $30 \pm 38$ days after surgery in Group B. The fistula completely disappeared in all patients. No recurrence of the fistula was noted in the follow-up period. 
TABLE 1. Summary of demographic data, radiological findings, treatment, and outcomes in 10 patients with spinal dural arteriovenous fistulas

\begin{tabular}{|c|c|c|c|c|c|c|c|c|c|c|}
\hline Case No. & $\begin{array}{c}\text { Age } \\
\text { (yrs)/Sex }\end{array}$ & $\begin{array}{l}\text { Level/ } \\
\text { Lesion }\end{array}$ & $\begin{array}{c}\text { Duration of } \\
\text { Symptoms } \\
\text { (mos) }\end{array}$ & $\begin{array}{c}\text { ALS Score }(G)^{*} \\
\text { Pre/ } \\
\text { Posttreatment } \dagger\end{array}$ & $\begin{array}{c}\text { ALS Score } \\
\text { (M) } \ddagger \text { Pre/ } \\
\text { Posttreatment }\end{array}$ & $\begin{array}{c}\text { Transit Time } \\
(\mathrm{sec})\end{array}$ & $\begin{array}{c}\text { No. of } \\
\text { Vertebral } \\
\text { Levels of T2- } \\
\text { HIA Pre/ } \\
\text { Posttreatment }\end{array}$ & Treatment & $\begin{array}{c}\text { Surgical } \\
\text { Complication }\end{array}$ & $\begin{array}{c}\text { Prophylactic } \\
\text { Low-Dose } \\
\text { Heparin }\end{array}$ \\
\hline \multicolumn{11}{|l|}{ Group A } \\
\hline 1 & $57 / \mathrm{M}$ & T6/dural & 12 & $4 / 4$ & $3 / 2$ & $\geq 13.0$ & $9 / 3$ & $S$ & - & - \\
\hline 2 & $39 / M$ & L1/dural & 31 & $3 / 2$ & $2 / 1$ & 12.3 & $8 / 4$ & $\mathrm{E} \rightarrow \mathrm{S} \S$ & $\begin{array}{l}\text { Subcutaneous } \\
\text { CSF fistula }\end{array}$ & - \\
\hline 3 & $77 / F$ & S/epidural & 45 & $4 / 4$ & $2 / 2$ & $\geq 11.3$ & $9 / 6$ & $S$ & - & + \\
\hline \multicolumn{11}{|l|}{ Group B } \\
\hline 4 & $55 / \mathrm{M}$ & T8/dural & 13 & $3 / 1$ & $1 / 0$ & NA & $9 / 0$ & $S$ & - & - \\
\hline 5 & $73 / \mathrm{M}$ & C1/dural & 60 & $4 / 3$ & $2 / 1$ & 5.0 & $6 / 1$ & $S$ & - & - \\
\hline 6 & $59 / \mathrm{M}$ & T7/dural & 36 & $2 / 1$ & $1 / 0$ & 6.5 & $4 / 0$ & $E \rightarrow S$ & - & - \\
\hline 7 & $53 / \mathrm{M}$ & T6/dural & 24 & $2 / 1$ & $1 / 0$ & 5.3 & $5 / 0$ & $E \rightarrow S$ & - & - \\
\hline 8 & $61 / \mathrm{M}$ & T7/dural & 27 & $1 / 1$ & $0 / 0$ & 8.3 & $1 / 0$ & $E \rightarrow S$ & - & - \\
\hline 9 & $56 / M$ & S/dural & 15 & $2 / 1$ & $1 / 0$ & 6.7 & $7 / 0$ & $S$ & - & - \\
\hline 10 & $57 / M$ & T6/dural & 27 & $5 / 4$ & $3 / 3$ & 5.5 & $1 / 0$ & $E \rightarrow S$ & - & - \\
\hline
\end{tabular}

$\mathrm{CSF}=$ cerebrospinal fluid.

Group A includes patients with acute postoperative neurological deterioration. Group B includes patients without acute postoperative deterioration.

* The ALS score for gait disturbance: grade 1 = leg weakness, no restriction of activity; grade 2 = reduced tolerance to exercise; grade $3=$ requires a cane; grade

$4=$ requires crutches or walker; grade $5=$ unable to stand, wheelchair user or in bed.

$\dagger$ Pre/posttreatment indicates at presentation and at 1 year after surgery.

‡ The ALS score for micturition dysfunction: grade 1 = hesitancy, frequency, urgency; grade 2 = occasional urinary incontinence or retention; grade $3=$ total incontinence or persistent retention.

$\S \mathrm{E} \rightarrow \mathrm{S}$ indicates surgery after failed endovascular embolization.

\section{Clinical Characteristics of Patients Who Presented With Acute Postoperative Neurological Deterioration}

The clinical presentation, treatment, and outcomes of 3 patients with acute deterioration are summarized in Table 2. Despite showing some improvement immediately after surgery, they developed severe myelopathy between postoperative days 1 and 3 . Neurological examination revealed severe sensorimotor deficits in both legs, including paraplegia with 1 to 3/5 muscle strength, severe sensory impairment of vibration and proprioception predominantly, and complete urinary retention. Because CT or MRI during exacerbation revealed no surgical complications, each patient received therapeutic dose of heparin for assumed progressive venous thrombosis. Three patients responded well to AC and improved to the level before exacerbation after several days. Because of neurological complications, these patients continued AC with an oral anticoagulant after 7 to 10 days of systemic heparinization.

Premature discontinuation of AC caused delayed deterioration in 2 patients. Patient 1 presented with gradual worsening of gait after discontinuing 3-month $\mathrm{AC}$, which is the protocol for postinterventional thrombosis. ${ }^{28}$ Patient 2 had rapidly progressive gait disturbance after discontinuation of AC when lumbar drain insertion to treat subcutaneous cerebrospinal fluid fistula was performed 1 month after surgery. MRI during exacerbation revealed severe spinal cord congestion similar to that before surgery, with some reduction of perimedullary flow voids. Reinstitution of systemic heparinization followed by the oral anticoagulant restored his symptoms to the level before exacerbation. Six months after surgery, Patient 2 developed delayed redeterioration after
AC was intentionally discontinued. MRI showed residual spinal cord congestion, predominantly in the conus medullaris, despite a marked reduction of perimedullary flow voids. After the resolution of spinal cord congestion was confirmed on follow-up MRI 12 months after surgery, discontinuing $A C$ no longer affected the neurological status. Eventually, Patient 2 showed a favorable outcome: the ALS scores for micturition and gait both decreased by one grade. In contrast, severe spinal ataxia related to this complication remained a sequela in Patients 1 and 3 , despite improved muscle weakness. Figure 1 shows DSA and follow-up MRI findings for Patient 2, the illustrative case of Group A.

\section{Comparison of Clinical and Radiological Data Between Groups}

Comparisons of clinical and radiological data between the groups are summarized in Table 3 . There were no significant differences in patient age $(p=0.852)$, duration of symptoms $(p=0.967)$, preoperative ALS scores for gait disturbance $(p=0.294)$, and micturition disor$\operatorname{der}(p=0.120)$ between the groups. However, Group A appeared to have more severe myelopathic symptoms before surgery than Group $B$, as evidenced by the large effect sizes for gait disturbance and micturition dysfunction ( $d=0.866$ and 1.342 , respectively).

On DSA, the mean transit time of the contrast medium was significantly higher in Group A than in Group B (12.2 $\pm 0.9 \mathrm{sec}$ and $6.2 \pm$ $1.2 \mathrm{sec}$, respectively; $p<0.001$ ) with an extremely large effect size $(d=6.001)$ and strong statistical power $([1-\beta]>0.999)$. In Patients 1 
TABLE 2. Clinical findings of patients who presented with acute neurological deterioration after surgical interruption of spinal dural arteriovenous fistulas

\begin{tabular}{|c|c|c|c|c|c|c|}
\hline Case No. & Age (yrs)/Sex & $\begin{array}{l}\text { Onset of Acute } \\
\text { Deterioration }\end{array}$ & Symptoms & $\begin{array}{c}\text { Delayed } \\
\text { Redeterioration } \\
\text { After Discontinuing } \\
\text { AC }\end{array}$ & Duration of $A C^{*}$ & $\begin{array}{c}\text { Permanent } \\
\text { Neurological } \\
\text { Deficit Related to } \\
\text { POND }\end{array}$ \\
\hline 1 & $57 / M$ & POD 3 & $\begin{array}{c}\text { Paraplegia }(1-3 / 5) \text {, } \\
\text { severe sensory } \\
\text { impairment, total } \\
\text { urinary retention }\end{array}$ & $\begin{array}{c}3 \text { mos after } \\
\text { surgery }\end{array}$ & $3 \mathrm{mos}$ & $\begin{array}{l}\text { Severe spinal } \\
\text { ataxia }\end{array}$ \\
\hline 2 & $39 / \mathrm{M}$ & POD 1 & $\begin{array}{c}\text { Paraplegia }(2-3 / 5) \text {, } \\
\text { severe sensory } \\
\text { impairment, total } \\
\text { urinary retention }\end{array}$ & $\begin{array}{c}1 \& 6 \text { mos after } \\
\text { surgery }\end{array}$ & $12 \mathrm{mos}$ & None \\
\hline 3 & $77 / F$ & POD 2 & $\begin{array}{c}\text { Paraplegia }(1-3 / 5) \\
\text { severe sensory } \\
\text { impairment, total } \\
\text { urinary retention }\end{array}$ & None & $18 \mathrm{mos}$ & $\begin{array}{l}\text { Severe spinal } \\
\text { ataxia }\end{array}$ \\
\hline
\end{tabular}

$\mathrm{POD}=$ postoperative day; POND = postoperative neurological deterioration.

* Anticoagulation therapy included intravenous continuous administration with therapeutic dose of heparin for 7 to 10 days initiated after acute deterioration followed by oral anticoagulant administration.

and 3 in Group $A$, the contrast medium stagnated within the measurement range in the last frame on DSA, underestimating each transit time.

The craniocaudal extent of preoperative intramedullary HIA ranged from 8 to 9 (mean, $8.7 \pm 0.6$ ) in Group $A$ and from 1 to 9 (mean, $4.7 \pm$ 3.0) in Group B. There was no significant difference between them $(p=0.059)$; however, spinal cord congestion was likely more extensive in Group A than in Group B because of an extremely large effect size $(d=1.699)$. The percent changes in intramedullary $\mathrm{HIA}$ at 3,6 , and 12 months after surgery were significantly lower in Group A than in Group B ( $p=0.026,0.002,<0.001$, respectively), with an extremely large effect size $(d=2.107$, $3.433,5.334$, respectively) and sufficiently strong statistical power $([1-\beta]=0.762,0.991,>0.999$, respectively $)$.

\section{Possible Predictors of Acute Postoperative Neurological Deterioration}

Acute deterioration was not significantly correlated with patient age or duration of symptoms (Table 4). Acute deterioration correlated
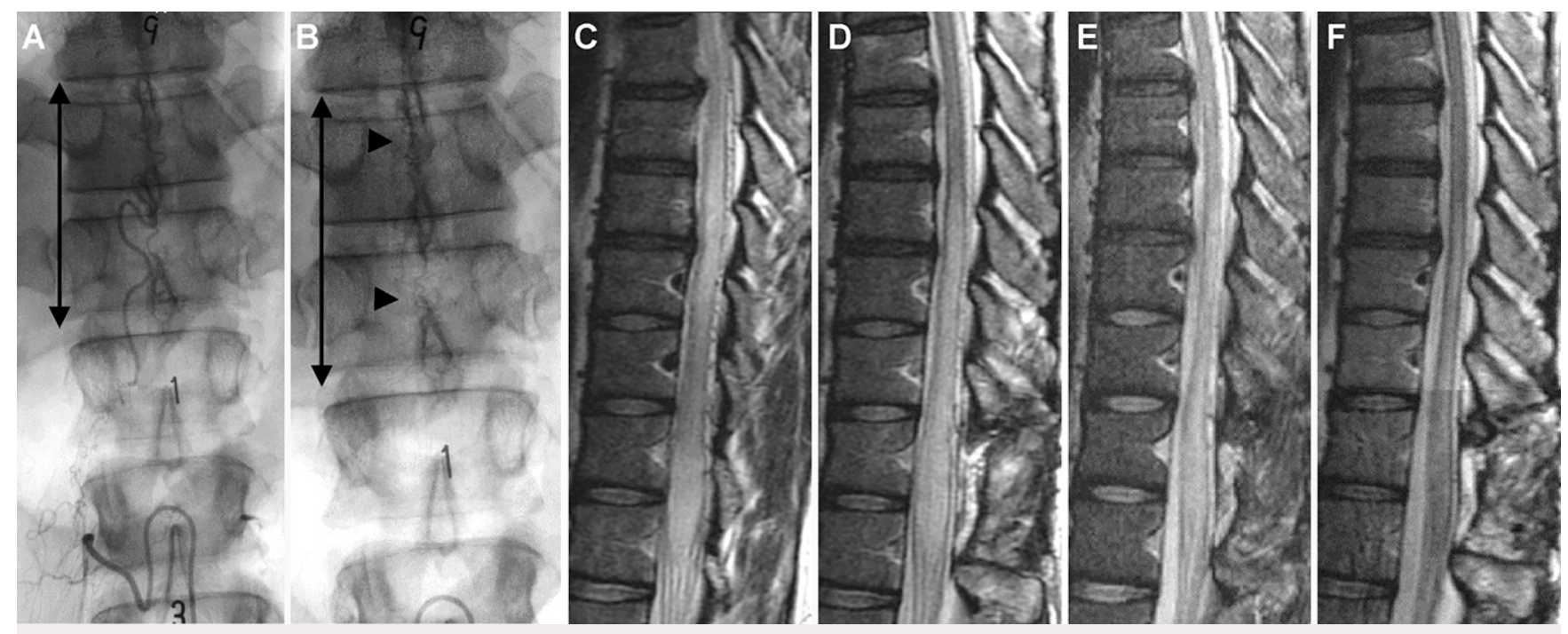

FIG. 1. Representative images of Patient 2 assigned to Group A. A: Anteroposterior angiogram obtained 9.0 seconds after injection of contrast medium presents a spinal dural arteriovenous fistula at the right L1 draining into the dilated perimedullary veins. Vertical arrow indicates the measurement range of the transit time between one level above and below the reflux point. B: The image obtained 16.5 seconds after injection presents the stagnated contrast medium (arrowhead) in the measurement range (vertical arrow). C: Preoperative T2-weighted MRI presents diffuse severe spinal cord congestion with perimedullary flow void sign. D: Image obtained 3 months after surgery shows persistent severe spinal cord congestion despite a gradual decrease in perimedullary flow voids. E: Image obtained on redeterioration after discontinuing anticoagulant 6 months after surgery shows persistent moderate spinal cord congestion and reduction of perimedullary flow voids. F: Image obtained 12 months after surgery shows decreased spinal cord congestion with mild swelling localized in the conus medullaris. 
TABLE 3. Comparison of clinical and radiological findings in patient groups

\begin{tabular}{|c|c|c|c|c|c|}
\hline Variable & Group A & Group B & p Value* & Cohen's d & $1-\beta \ddagger$ \\
\hline Age, yrs & $57.7 \pm 19.0$ & $59.1 \pm 6.6$ & 0.852 & 0.149 & 0.054 \\
\hline ALS score $(G)$ at presentation & $3.7 \pm 0.6$ & $2.7 \pm 1.4$ & 0.294 & 0.866 & 0.198 \\
\hline ALS score $(\mathrm{M})$ at presentation & $2.3 \pm 0.6$ & $1.3 \pm 1.0$ & 0.120 & 1.342 & 0.402 \\
\hline \multicolumn{6}{|l|}{ Radiological data } \\
\hline Transit time, sec & $12.2 \pm 0.9$ & $6.2 \pm 1.2$ & $<0.001$ & 6.001 & $>0.999$ \\
\hline No. of vertebral levels of HIA on T2-weighted MRI before surgery & $8.7 \pm 0.6$ & $4.7 \pm 3.0$ & 0.059 & 1.699 & 0.580 \\
\hline \multicolumn{6}{|l|}{ Percent changes in intramedullary HIA§, \% } \\
\hline
\end{tabular}

$\mathrm{F} / \mathrm{U}=$ follow-up.

All data are expressed as means \pm standard deviations.

$* p$ value for significance is set at 0.05 .

$\dagger$ A value of Cohen's $d$ is defined by conversion as follows: $d=0.2$, small effect size; 0.5 , medium effect size; 0.8 , large effect size; $>1.0$, extremely large effect size.

$\ddagger A$ value of $(1-\beta)$ for strong statistical power is 0.95 under the significance level set at 0.05 .

$\S$ Percent changes in intramedullary HIA $(\%)=$ (number of vertebral levels of intramedullary HIA on follow-up MRI - number of vertebral levels of intramedullary HIA on preoperative MRI) $\times 100 /$ number of vertebral levels of intramedullary HIA on preoperative MRI.

weakly and moderately with the ALS for gait disturbance and micturition dysfunction, respectively (coefficient $=0.391,0.558$, respectively); however, these differences were not statistically significant $(p=0.264$, 0.094 , respectively). The transit time had a very strong positive correlation with acute deterioration (coefficient $=0.825 ; p=0.006$ ). The extent of intramedullary HIA on preoperative MRI had a strong positive correlation (coefficient $=0.656 ; p=0.040$ ).

\section{Discussion}

\section{Observations}

Acute neurological deterioration after SDAVF treatment is an uncommon complication but a clinically critical management challenge that can worsen a patient's functional outcomes. In our study, we have shown that a significant circulation delay on preoperative
DSA and prominent spinal cord congestion on preoperative MRI may be predictors for this complication. In addition, we clarified the therapeutic setbacks pertaining to this complication based on the treatment of severe cases.

Currently, unexpected venous thrombosis of the spinal cord after SDAVF treatment is considered the main cause of acute deterioration. ${ }^{17-23,25}$ This study supports this hypothesis with the following results: (1) AC was efficacious in treating acute deterioration, and discontinuation and reinstitution of $A C$ directly affected redeterioration and improvement of the symptoms, respectively; (2) acute deterioration developed with a delay of 1 to 3 days after surgery; and (3) the dorsal spinal cord was primarily involved, in which dilated perimedullary veins were predominantly present on its surface. $^{29}$

\section{TABLE 4. Results of Spearman's rank correlation between postoperative neurological deterioration and each variable}

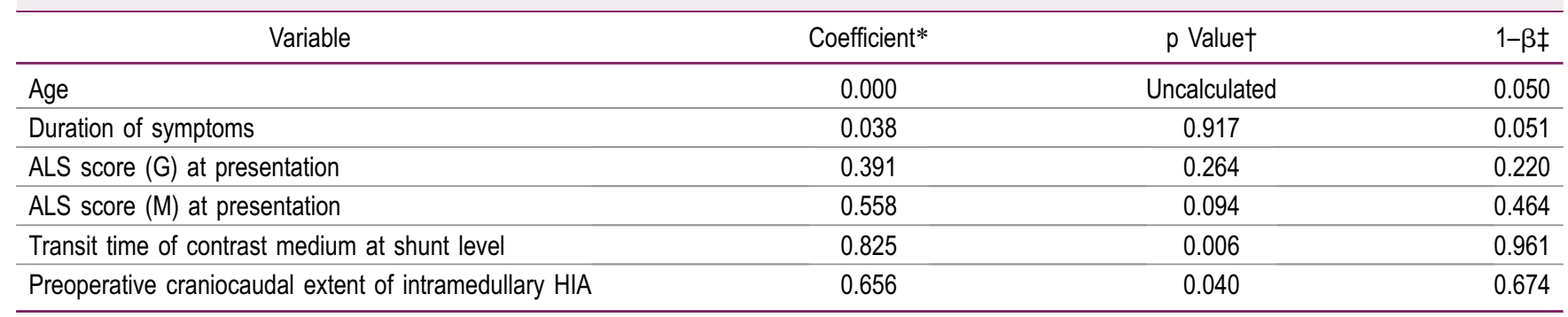

* The strength of the correlation was defined as follows: Coefficient: 0.20 to 0.39 , weak correlation; 0.40 to 0.59 , moderate correlation; 0.60 to 0.79 , strong correlation; 0.80 to 1.0 , very strong correlation.

$\dagger p$ value for significance is set at 0.05 .

$\ddagger A$ value of $(1-\beta)$ for strong statistical power is 0.95 under the significance level set at 0.05 . 
Our literature search revealed that the frequency of such occurrence was $4.3 \%$ to $32.0 \%$, regardless of the therapeutic modality used. ${ }^{17-24,30-32}$ This complication has been reported as a reversible process in most patients. ${ }^{17-23,30-32}$ However, unlike the findings of previous studies, our study reports that in 2 of the 3 patients with acute deterioration, this complication caused permanent neurological deficit and worsened the functional outcomes. This finding suggested that fistula obliteration may have a paradoxical effect on some patients, resulting in poor functional outcomes.

A study by Hassler et al. ${ }^{25}$ showed the clinical significance of intraoperative hemodynamics in SDAVF for predicting high risk of developing acute postoperative deterioration. They observed that in a low-flow fistula, which exhibited the highest venous outflow resistance, dilated perimedullary veins did not collapse because of the highest residual venous pressure after shunt obliteration. Additionally, Doppler sonography revealed that these veins lost detectable flow signals. These observations led them to speculate that uncollapsed and stagnating veins after shunt obliteration were more likely to develop progressive thrombosis, explaining the transient postoperative neurological deterioration. In our study, all patients with acute deterioration had a significantly extended transit time of the contrast medium. These patients can be regarded as illustrative cases supporting the hypothesis by Hassler et al. The transit time, which showed a strong positive correlation with acute deterioration, may be considered a strong predictor. Therefore, it is essential to evaluate the degree of venous outflow impairment on DSA for predicting high risk of postoperative deterioration before surgery.

The clinical significance of intramedullary HIA on preoperative MRI as a predictor of acute deterioration needs to be considered in association with venous outflow impairment. Some authors have shown a strong positive correlation between neurological status, extent of dilated perimedullary veins on DSA, and extent of spinal cord congestion on $\mathrm{MRI}^{33,34}$ As the stage of the disease progresses, radicular veins near the fistula, which act as a venous decompression point in the early stage of the disease, become progressively obstructed. Elevated pressure in perimedullary veins directly affects intramedullary veins until it is reduced by a venous decompression point far from the fistula, resulting in congestive spinal cord edema along the enlarged perimedullary veins. $^{33,34}$ In patients with extensive spinal cord edema, if the function of the venous decompression point is preserved, the risk of acute deterioration is low; reduced venous drainage from the venous decompression points increases the risk.

Comparing chronological changes in patients with and without acute deterioration with follow-up MRI is important to elucidate the nature of this complication. The most characteristic finding in Group A was a significant delay in the reduction of spinal cord congestion. A time-dependent reduction in spinal cord signal abnormalities after treatment reflects an improvement in venous circulation of the spinal cord. ${ }^{35-38}$ On the other hand, prolonged spinal cord signal abnormalities indicate irreversible changes in the spinal cord or the recurrent arteriovenous shunt, especially in cases with insufficient improvement in perimedullary flow void sign. ${ }^{35-38}$ In this study, postoperative DSA confirmed the complete disappearance of fistulas, and follow-up MRIs revealed gradual reduction of perimedullary flow voids time dependently in all patients with acute deterioration; therefore, prolonged spinal cord congestion presumably reflected the exacerbated venous circulation due to posttreatment venous thrombosis in addition to the preexisting severe venous outflow obstruction.

The optimal duration of $\mathrm{AC}$ for progressive venous thrombosis after SDAVF treatment has not yet been established. Ohta et al. reported the case of a patient with postinterventional thrombosis requiring dual antiplatelet therapy for a year. ${ }^{20}$ In that study, after discontinuing AC, delayed deterioration was observed even at 6 months after surgery. These findings indicate that patients with severe venous outflow impairment may be susceptible to posttreatment venous thrombosis for $\geq 6$ months. In a review of thrombosis of the cerebral veins and sinuses, Stam suggested that administration of vitamin $\mathrm{K}$ antagonists should be continued for 6 months after the first episode of sinus thrombosis or be extended further in the presence of predisposing factors. ${ }^{39}$ This study revealed that discontinuing anticoagulants with residual severe spinal cord congestion caused delayed deterioration, indicating that severe spinal cord congestion may be a predisposing factor of posttreatment venous thrombosis in patients with acute deterioration.

\section{Lessons}

Acute neurological deterioration after SDAVF treatment is more likely to develop in patients with severe venous outflow impairment. This development is due to prolonged spinal cord congestion caused by postoperative progressive venous thrombosis and the preexistence of severe venous outflow impairment. AC should be continued for patients with acute deterioration until the resolution of spinal cord congestion is confirmed by follow-up MRI.

This study has several limitations. First, owing to the rarity of the complication, the sample size was small. In the differences in the patients' backgrounds, neurological status before and after surgery, and the degree of spinal cord congestion on MRI before surgery, the statistical power was limited because of small sample size. However, circulation delay on DSA and prolonged spinal cord congestion were detected with statistical significance and strong statistical power. Second, we could not clarify the indication and efficacy of heparin prophylaxis to prevent acute deterioration in this retrospective study. One patient who received low-dose prophylactic heparin presented with acute deterioration, indicating that this heparin dosage was probably insufficient to suppress the thrombotic process. A similar phenomenon has also been reported by Jablawi et al. ${ }^{22}$ A prospective study on heparin prophylaxis in cohorts classified based on the degree of venous outflow impairment on DSA is warranted. Assessing a larger cohort of patients with SDAVF may reveal the characteristics of this unfavorable phenomenon and importance of prophylactic heparinization, with more statistical power, and thus contribute to improving functional outcomes.

\section{Acknowledgments}

We are grateful to Drs. Y. Ito and K. Nishino, Department of Neurosurgery, Brain Research Institute, Niigata University, for their expert care in endovascular management in diagnosing and treating SDAVF. We are also grateful to Drs. M. Fukuda and M. Oishi, Department of Neurosurgery, Brain Research Institute, Niigata University, for intraoperative support with electrophysiological monitoring.

\section{References}

1. Hurst RW, Kenyon LC, Lavi E, Raps EC, Marcotte P. Spinal dural arteriovenous fistula: the pathology of venous hypertensive myelopathy. Neurology. 1995;45(7):1309-1313.

2. Kataoka H, Miyamoto S, Nagata I, Ueba T, Hashimoto N. Venous congestion is a major cause of neurological deterioration in spinal arteriovenous malformations. Neurosurgery. 2001;48(6):1224-1230.

3. Jellema K, Tijssen CC, van Gijn J. Spinal dural arteriovenous fistulas: a congestive myelopathy that initially mimics a peripheral nerve disorder. Brain. 2006;129(Pt 12):3150-3164. 
4. Tender GC, Vortmeyer AO, Oldfield EH. Spinal intradural arteriovenous fistulas acquired in late adulthood: absent spinal venous drainage in pathogenesis and pathophysiology. Report of two cases. $J$ Neurosurg Spine. 2005;3(6):488-494.

5. Geibprasert S, Pereira V, Krings T, et al. Dural arteriovenous shunts: a new classification of craniospinal epidural venous anatomical bases and clinical correlations. Stroke. 2008;39(10):2783-2794.

6 . Sato K, Terbrugge KG, Krings T. Asymptomatic spinal dural arteriovenous fistulas: pathomechanical considerations. J Neurosurg Spine. 2012;16(5):441-446.

7. Kimura A, Tan CF, Wakida K, et al. Venous congestive myelopathy of the cervical spinal cord: an autopsy case showing a rapidly progressive clinical course. Neuropathology. 2007;27(3):284-289.

8. Rodriguez FJ, Crum BA, Krauss WE, Scheithauer BW, Giannini C. Venous congestive myelopathy: a mimic of neoplasia. Mod Pathol. 2005;18(5):710-718.

9. McCutcheon IE, Doppman JL, Oldfield EH. Microvascular anatomy of dural arteriovenous abnormalities of the spine: a microangiographic study. J Neurosurg. 1996;84(2):215-220.

10. Takai K, Komori T, Taniguchi M. Microvascular anatomy of spinal dural arteriovenous fistulas: arteriovenous connections and their relationships with the dura mater. J Neurosurg Spine. 2015;23(4):526-533.

11. Afshar JK, Doppman JL, Oldfield EH. Surgical interruption of intradural draining vein as curative treatment of spinal dural arteriovenous fistulas. J Neurosurg. 1995;82(2):196-200.

12. Jellema K, Sluzewski M, van Rooij WJ, Tijssen CC, Beute GN. Embolization of spinal dural arteriovenous fistulas: importance of occlusion of the draining vein. J Neurosurg Spine. 2005;2(5):580-583.

13. Koch MJ, Stapleton CJ, Agarwalla PK, et al. Open and endovascular treatment of spinal dural arteriovenous fistulas: a 10-year experience. J Neurosurg Spine. 2017;26(4):519-523.

14. Steinmetz MP, Chow MM, Krishnaney AA, et al. Outcome after the treatment of spinal dural arteriovenous fistulae: a contemporary singleinstitution series and meta-analysis. Neurosurgery. 2004;55(1):77-88.

15. Narvid J, Hetts SW, Larsen D, et al. Spinal dural arteriovenous fistulae: clinical features and long-term results. Neurosurgery. 2008; 62(1):159-167.

16. Bretonnier M, Hénaux PL, Gaberel T, et al. Spinal dural arteriovenous fistulas: clinical outcome after surgery versus embolization: a retrospective study. World Neurosurg. 2019;127:e943-e949.

17. Huffmann BC, Gilsbach JM, Thron A. Spinal dural arteriovenous fistulas: a plea for neurosurgical treatment. Acta Neurochir (Wien). 1995;135(1-2):44-51.

18. Westphal $M$, Koch $C$. Management of spinal dural arteriovenous fistulae using an interdisciplinary neuroradiological/neurosurgical approach: experience with 47 cases. Neurosurger8.

19. Niimi Y, Berenstein A, Setton A, Neophytides A. Embolization of spinal dural arteriovenous fistulae: results and follow-up. Neurosurgery. 1997;40(4):675-683.

20. Ohta T, Gomi M, Oowaki H, Ishikawa M. Chronic venous congestion following embolization of spinal dural fistula. Case report. $J$ Neurosurg Spine. 2008;9(2):186-190.

21. Knopman J, Zink W, Patsalides A, Riina HA, Gobin YP. Secondary clinical deterioration after successful embolization of a spinal dural arteriovenous fistula: a plea for prophylactic anticoagulation. Interv Neuroradiol. 2010;16(2):199-203.

22. Jablawi F, Schubert GA, Hans FJ, Mull M. Anticoagulation therapy after surgical treatment of spinal dural arteriovenous fistula. Effectiveness and long-term outcome analysis. World Neurosurg. 2018;114:e698-e705.

23. Ushikoshi S, Hida K, Kikuchi Y, Miyasaka K, Iwasaki T, Abe H. Functional prognosis after treatment of spinal dural arteriovenous fistulas. Neurol Med Chir (Tokyo). 1999;39(3):206-213.
24. Saladino A, Atkinson JL, Rabinstein AA, et al. Surgical treatment of spinal dural arteriovenous fistulae: a consecutive series of 154 patients. Neurosurgery. 2010;67(5):1350-1358.

25. Hassler W, Thron A, Grote EH. Hemodynamics of spinal dural arteriovenous fistulas. An intraoperative study. J Neurosurg. 1989;70(3):360-370.

26. Aminoff MJ, Logue $\mathrm{V}$. The prognosis of patients with spinal vascular malformations. Brain. 1974;97(1):211-218.

27. Burkhardt JK, Safaee MM, Clark AJ, Lawton MT. Sacral epidural arteriovenous fistulas: imitators of spinal dural arteriovenous fistulas with different pathologic anatomy: report of three cases and review of the literature. Acta Neurochir (Wien). 2017;159(6):1087-1092.

28. Casasco AE, Houdart E, Jhaveri HS, et al. Embolization of spinal vascular malformations. In: Connors JJ III and Wojak JC, ed. Interventional Neuroradiology: Strategies and Practical Techniques. W. B. Saunders; 1999:186-198.

29. Krings T, Geibprasert S. Spinal dural arteriovenous fistulas. AJNR Am J Neuroradiol. 2009;30(4):639-648.

30. Mourier KL, Gelbert F, Rey A, et al. Spinal dural arteriovenous malformations with perimedullary drainage. Indications and results of surgery in 30 cases. Acta Neurochir (Wien). 1989;100(3-4):136-141.

31. Tacconi L, Lopez Izquierdo BC, Symon L. Outcome and prognostic factors in the surgical treatment of spinal dural arteriovenous fistulas. A long-term study. Br J Neurosurg. 1997;11(4):298-305.

32. Cecchi PC, Musumeci A, Rizzo P, Faccioli F, Bricolo A. Late deterioration of neurologic function in patients surgically treated for spinal dural arteriovenous fistulas. Surg Neurol. 2009;72(3):257-262.

33. Yen PP, Ritchie KC, Shankar JJ. Spinal dural arteriovenous fistula: correlation between radiological and clinical findings. J Neurosurg Spine. 2014;21(5):837-842.

34. Hetts SW, Moftakhar P, English JD, et al. Spinal dural arteriovenous fistulas and intrathecal venous drainage: correlation between digital subtraction angiography, magnetic resonance imaging, and clinical findings. J Neurosurg Spine. 2012;16(5):433-440.

35. Willinsky RA, terBrugge K, Montanera W, Mikulis D, Wallace MC. Posttreatment MR findings in spinal dural arteriovenous malformations. AJNR Am J Neuroradiol. 1995;16(10):2063-2071.

36. Horikoshi T, Hida K, Iwasaki Y, Abe H, Akino M. Chronological changes in MRI findings of spinal dural arteriovenous fistula. Surg Neurol. 2000;53(3):243-249.

37. Isu T, Iwasaki Y, Akino M, Koyanagi I, Abe H. Magnetic resonance imaging in cases of spinal dural arteriovenous malformation. Neurosurgery. 1989;24(6):919-923.

38. Kaufmann TJ, Morris JM, Saladino A, Mandrekar JN, Lanzino G. Magnetic resonance imaging findings in treated spinal dural arteriovenous fistulas: lack of correlation with clinical outcomes. J Neurosurg Spine. 2011;14(4):548-554.

39. Stam J. Thrombosis of the cerebral veins and sinuses. N Engl J Med. 2005;352(17):1791-1798.

\section{Disclosures}

The authors report no conflict of interest concerning the materials or methods used in this study or the findings specified in this paper.

\section{Author Contributions}

Conception and design: all authors. Acquisition of data: all authors. Analysis and interpretation of data: all authors. Drafting the article: Saito, Fujii. Critically revising the article: Saito. Reviewed submitted version of manuscript: all authors. Approved the final version of the manuscript on behalf of all authors: Saito. Statistical analysis: Saito, Yajima, Nakamura. Administrative/technical/material support: Saito, Yajima, Nakamura. Study supervision: Fujii.

\section{Correspondence}

Akihiko Saito: Niigata City General Hospital, Niigata, Japan. akisaito@ bri.niigata-u.ac.jp. 